\title{
Planetary Defence Activities Beyond NASA and ESA
}

\author{
Brent W. Barbee
}

\section{Introduction}

The collision of a significant asteroid or comet with Earth represents a singular natural disaster for a myriad of reasons, including: its extraterrestrial origin; the fact that it is perhaps the only natural disaster that is preventable in many cases, given sufficient preparation and warning; its scope, which ranges from damaging a city to an extinction-level event; and the duality of asteroids and comets themselves---they are grave potential threats, but are also tantalising scientific clues to our ancient past and resources with which we may one day build a prosperous spacefaring future.

Accordingly, the problems of developing the means to interact with asteroids and comets for purposes of defence, scientific study, exploration, and resource utilisation have grown in importance over the past several decades. Since the 1980s, more and more asteroids and comets (especially the former) have been discovered, radically changing our picture of the solar system. At the beginning of the year 1980, approximately 9,000 asteroids were known to exist. By the beginning of 2001, that number had risen to approximately 125,000 thanks to the Earth-based telescopic survey efforts of the era, particularly the emergence of modern automated telescopic search systems, pioneered by the Massachusetts Institute of Technology's (MIT's) LINEAR system in the mid-to-late 1990s. ${ }^{1}$ Today, in late 2019, about 840,000 asteroids have been discovered, ${ }^{2}$ with more and more being found every week, month, and year. Of those, approximately 21,400 are categorised as near-Earth asteroids (NEAs), 2,000 of which are categorised as Potentially Hazardous Asteroids (PHAs) ${ }^{3}$ and 2,749 of which are categorised as potentially accessible. ${ }^{4}$

The hazards posed to us by asteroids affect people everywhere around the world. As well, the opportunities presented by asteroids may benefit our entire species. Thus, with such a large number of currently known asteroids and so many yet to be discovered, it is not surprising that individuals, organisations, institutions, and governments all around the world have become interested in the study of asteroids. Indeed, a variety of

\footnotetext{
${ }^{1}$ Scott Manley, 'Asteroid Discovery - 1970-2015 - 8K resolution' (YouTube, 24 September 2015) $<$ https://www.youtube.com/watch?v=BKKg4lZ_o-Y> accessed 2 December 2019

2 'JPL Small-Body Database Search Engine' $<$ https://ssd.jpl.nasa.gov/sbdb_query.cgi $>$ accessed 2 December 2019

3 'Discovery Statistics' $<$ https://cneos.jpl.nasa.gov/stats/totals.html $>$ accessed 8 November 2019

4 'Accessible NEAs' <https://cneos.jpl.nasa.gov/nhats/> accessed 8 November 2019; Note that NASA's Near-Earth Object Human Space Flight Accessible Targets Study (NHATS) uses a set of spacecraft mission design criteria for categorising NEAs as "NHATS-compliant." i.e., "potentially accessible." These criteria are documented at $<$ https://cneos.jpl.nasa.gov/nhats/caveats.html > accessed 26 December 2019, which include that the NEA must offer at least one round-trip mission opportunity between 2020 and 2045 for which the total propulsive change-in-velocity required is $\leq 12 \mathrm{~km} / \mathrm{s}$, the Earth departure 'characteristic energy' (typically denoted as ' $\mathrm{C}_{3}$ ') is $\leq 60 \mathrm{~km}^{2} / \mathrm{s}^{2}$, the roundtrip mission duration is $\leq 450$ days, at least 8 days are spent rendezvoused with the NEA, and the Earth return atmospheric entry speed (propulsively controlled when necessary) is $\leq 12 \mathrm{~km} / \mathrm{s}$.
} 
government space agencies, private organisations, and individuals have worked on developing the means by which to observe, study, and even interact with asteroids and comets for purposes including science, exploration, pioneering, commerce, and planetary defence. This includes significant individual contributions by amateur asteroid astronomers all over the world. International cooperation in planetary defence within the contexts of the United Nations and the International Asteroid Warning Network (IAWN) are discussed in Chapter 2, and the activities undertaken by the world's larger space agencies, ESA and NASA, are discussed in Chapters 3 and 4. But, what of the other agencies and institutions around the world who are also working on the problem of defence against hazardous asteroids and comets, or related topics? In this chapter we provide an overview, in alphabetical order, of some of the planetary defence related efforts that have been undertaken around the world beyond the activities at the United Nations, NASA, and ESA.

\section{B612 Foundation}

The B612 Foundation is a private non-profit foundation (501(c)(3) organisation) whose headquarters is located in Mill Valley, California, USA. B612 was founded in 2002 by Ed Lu, Rusty Schweickart, Piet Hut, and Clark Chapman, with the goal of "significantly altering the orbit of an asteroid in a controlled manner." The foundation is named after the asteroid lived on by the main character of the 1943 novella entitled The Little Prince. B612 has previously advocated for increased NASA funding to enable completion of the search for potentially hazardous near-Earth objects (NEOs) and worked to help motivate and organise international planetary defence preparedness efforts. In 2012, ten years after its founding, B612 began attempting to raise private donation funding to deploy an infrared space-based NEO survey telescope mission named Sentinel. ${ }^{5}$ The mission was originally planned to launch in 2017 or 2018, but by 2015 efforts to raise funds for the mission were falling short of the needed funding milestones. ${ }^{6}$ As a result, B612 decided to end development of Sentinel.

After discontinuing Sentinel development, B612 began studying the use of the synthetic tracking technique deployed on multiple smaller telescope spacecraft as an alternative concept to the more traditional single, large telescope spacecraft. ${ }^{7}$ The foundation's focus then shifted to algorithm development and data analysis, and those efforts continue as of the time of this writing. In 2018, B612 announced the consolidation of their research efforts under the Asteroid Institute, a virtual organisation collaborating with the Data Intensive

\footnotetext{
${ }^{5}$ Debra Werner, 'Group Raising Funds for Telescope To Detect Deadly Asteroids' (SpaceNews 9 July 2012) < https://spacenews.com/group-raising-funds-telescope-detect-deadly-asteroids/> accessed 21 November 2019; Note that Sentinel was intended to be an infrared space-based NEO survey telescope placed in a 'Venus-trailing' or 'Venus-like' orbit, i.e., an orbit around the Sun similar to Venus' orbit, but with the spacecraft not located near Venus itself.

${ }^{6}$ Traci Watson, 'Private asteroid hunt lacks cash to spy threats in orbit' (Nature 19 June 2015) $<$ https://www.nature.com/news/private-asteroid-hunt-lacks-cash-to-spy-threats-in-orbit-1.17810> accessed 21 November 2019

${ }^{7}$ Jeff Foust, 'B612 studying smallsat missions to search for near Earth objects' (Spacenews, 20 June 2017) < https://spacenews.com/b612-studying-smallsat-missions-to-search-for-near-earth-objects/> accessed 21 November 2019
} 
Research in Astrophysics and Cosmology Center (DIRAC) at the Department of Astronomy of the University of Washington. ${ }^{8}$ One of their analysis projects is the Asteroid Decision Analysis Machine (ADAM), the goal of which is to improve abilities to make decisions on potential asteroid threats.

\section{Canada}

The Canadian Space Agency (CSA) and Defence Research and Development Canada (DRDC) funded the development and launch of a small Canadian spacecraft named NEOSSat (Near Earth Object Surveillance Satellite). ${ }^{9}$ NEOSSat was originally scheduled to launch in 2007, but the launch was delayed until February of 2013. The goal of the $74 \mathrm{~kg}$ spacecraft was the detection of NEOs, particularly those with orbits largely or entirely interior to Earth's orbit around the Sun.

However, various problems that included issues with the electrical system, camera control software, and pointing stability systems degraded the quality of images produced by the spacecraft, which prevented it from being able to carry out its planned NEO detection mission. ${ }^{10}$ The issues preventing NEOSSat from operating as intended are detailed in the report of an audit conducted by the CSA's Audit and Evaluation Directorate, which was published in early $2014 .{ }^{11}$

\section{China (CNSA)}

\subsection{Chang'e 2 Flyby of Potentially Hazardous Near-Earth Asteroid 4179 Toutatis (1989 AC)}

The China National Space Administration (CNSA) launched the Chang'e 2 spacecraft on October 1st, 2010, after which it proceeded to enter orbit around the Moon and begin mapping the lunar surface. The spacecraft's primary mission objectives were completed by November of 2010, and detailed lunar surface maps constructed using data collected by the spacecraft were published during the months and years that followed. Meanwhile, the spacecraft was directed to depart lunar orbit in June 2011 and travel to the Sun-Earth Lagrange point 2 (SEL2), where it arrived in August 2011. In doing so, it became the first spacecraft ever to travel directly from lunar orbit to SEL2. Chang'e 2 was then directed to depart SEL2 in April of 2012 and proceed to a flyby

\footnotetext{
8 'B612 Annual Progress Report 2018'<https://b612foundation.org/wp-content/uploads/2018/10/2018-B612Annual-Progress-Report.pdf $>$ accessed 21 November 2019

9 'NEOSSat: Canada's Sentinel in the Sky'<http://www.asc-csa.gc.ca/eng/satellites/neossat/> accessed 21 November 2019

${ }^{10}$ Jordan Press, 'Asteroid-tracking satellite not up to the job: review' (Ottawa Citizen, 6 July 2014) $<$ https://ottawacitizen.com/news/national/asteroid-tracking-satellite-not-up-to-the-job-review $>$ accessed 21 November 2019

${ }^{11}$ Canadian Space Agency, Evaluation of the Near Earth Object Surveillance Satellite (NEOSSat) Project For the period from February 2005 to December 2013, Project \# 13/14 02-02, Prepared by the Audit and Evaluation Directorate, February 2014
} 
of the PHA 4179 Toutatis (1989 AC) on December 13th, 2012, when the asteroid was approximately 7 million $\mathrm{km}$ from Earth.

Toutatis is categorised as a PHA due to its Earth minimum orbit intersection distance (MOID) of 0.0064 $\mathrm{au}$ (astronomical units; $1 \mathrm{au}$ is the mean distance of Earth from the Sun, 149,597,870.7 km). The asteroid has a heliocentric orbit semi-major axis of $2.543 \mathrm{au}$, eccentricity of 0.626 , and inclination of $0.44^{\circ}$. Its absolute magnitude is 15.3 and its albedo is 0.405 , which together suggest an approximate diameter of 1,800 m. By contrast, planetary radar observations revealed that Toutatis is rather irregularly shaped, with dynamically equivalent, equal volume ellipsoid dimensions of 1,700 $\mathrm{m} \times 2,030 \mathrm{~m} \times 4,260 \mathrm{~m}$. Lightcurve observations show its rotation period to be 176 hours. ${ }^{12}$ Thus, Toutatis serves as a good example of how challenging it can be to assess an asteroid's true shape and size without radar imagery and/or close proximity imaging via spacecraft.

The Chang'e 2 spacecraft passed within $3.2 \mathrm{~km}$ of Toutatis at a relative velocity of $10.73 \mathrm{~km} / \mathrm{s}$ and collected images of the asteroid at approximately 10 meter per pixel resolution. The spacecraft imagery was later compared to the radar-derived shape model of Toutatis, revealing some small but interesting differences between the radar-derived shape model and the actual asteroid shape shown in the spacecraft camera images. ${ }^{13}$ With the completion of the Toutatis flyby, China became the fourth spaceflight-capable nation to deploy a successful mission to an asteroid, following NASA, ESA, and the Japan Aerospace Exploration Agency (JAXA).

\subsection{Potential Mission to 469219 Kamo`oalewa $\left(2016 \mathrm{HO}_{3}\right)$}

In April of 2019, CNSA announced a concept for a ten-year mission named ZhengHe, which would launch in 2022 or 2024 to collect and return samples of the NEA 469219 Kamo'oalewa $\left(2016 \mathrm{HO}_{3}\right)$, which is also categorised as an Earth quasi-satellite. Although not captured by the Earth's gravity, the asteroid makes distant repeating loops around the Earth when viewed in a frame of reference that moves with Earth along its orbit around the Sun. The repeating loop-like motion around Earth, which occurs well beyond the Earth's gravitational sphere of influence, is due to the nature of the relative motion between the Earth and the asteroid as they move independently around the Sun.

Kamo'oalewa has an Earth MOID of 0.0333 au, which meets the PHA Earth MOID criterion of less than or equal to $0.05 \mathrm{au}$. However, the asteroid is not technically categorised as a PHA because its absolute magnitude of 24.3 does not meet the PHA criterion of absolute magnitude less than or equal to 22.0. An absolute magnitude less than or equal to 22 corresponds to an approximate physical size of $140 \mathrm{~m}$ or larger, assuming an albedo (surface reflectivity) of approximately 14\%. The asteroid has a heliocentric orbit semi-major axis of $1.0011 \mathrm{au}$, eccentricity of 0.1035 , and inclination of $7.78^{\circ}$. Its absolute magnitude suggests that its approximate

\footnotetext{
12 '4179 Toutatis (1989 AC)' (Jet Propulsion Laboratory Small-Body Database Browser) $<$ https://ssd.jpl.nasa.gov/sbdb.cgi?sstr=Toutatis $>$ accessed 25 November 2019

${ }^{13}$ Xiaoduan Zou, et al., 'The preliminary analysis of the 4179 Toutatis snapshots of the Chang'E-2 flyby,' Icarus, Volume 229, Feb 2014, pp. 348-354
} 
size should be between $23 \mathrm{~m}$ and $105 \mathrm{~m}$, depending on how reflective the asteroid's surface is. Lightcurve observations show its rotation period to be 0.476 hours. ${ }^{14}$

After collecting samples from Kamo'oalewa and returning them to Earth, the ZhengHe spacecraft would then proceed to the main asteroid belt region beyond the orbit of Mars, where it would visit and study the object 7968 Elst-Pizarro, which is categorised as both an asteroid (due to its orbital location within the solar system) and a comet (due to its display of a cometary dust tail when near perihelion). As with most missions to asteroids or comets, the goals of ZhengHe would include studying the compositions and origins of the solar system, as well as the origins of life on Earth. ${ }^{15}$ As of the time of this writing, the Chinese government has not yet formally approved the ZhengHe mission.

\section{Denmark}

A group called the Emergency Asteroid Defence Project (EADP) formed in Denmark circa 2015, with the goal of crowdfunding a standing NEO deflection system based on the Hypervelocity Asteroid Intercept Vehicle (HAIV) concept. ${ }^{16}$ The HAIV concept was developed by Professor Bong Wie and his graduate students at the Asteroid Deflection Research Collaboration (ADRC), located in the Department of Aerospace Engineering at Iowa State University. The EADP website ${ }^{17}$ does not appear to have been updated since 2015, and there are no indications that the project was able to move forward. Their Indiegogo crowdfunding website for collecting donations indicates that they were able to raise $\$ 8,834$ USD, from 187 donors ("backers"), towards their original goal of $\$ 200,000$ USD. $^{18}$

The EADP concept of collecting private donations to fund a non-governmental planetary defence related initiative is unique thus far, with only the B612 Foundation's attempt to fund an NEO survey telescope using private donations being comparable. The inability of either the EADP or B612 efforts to ultimately fund their missions and bring them to fruition suggests that planetary defence mission activities will remain the province of governments, at least for the time being. However, the fact that some donor funds were able to be raised at all is still significant, demonstrating that some citizens are interested in planetary defence and believe that conducting planetary defence activities is an important and worthwhile use of resources.

\section{Japan (JAXA)}

\footnotetext{
14 '469219 Kamo`oalewa (2016 HO3)' (Jet Propulsion Laboratory Small-Body Database Browser) $<$ https://ssd.jpl.nasa.gov/sbdb.cgi?sstr=2016HO3> accessed 25 November 2019

${ }^{15}$ Elizabeth Gibney, 'China plans mission to Earth's pet asteroid' (Nature, 30 April 2019) $<$ https://www.nature.com/articles/d41586-019-01390-5> accessed 21 November 2019

${ }^{16}$ Brent Barbee, et al, 'Conceptual design of a flight validation mission for a Hypervelocity Asteroid Intercept Vehicle’ Acta Astronautica, Vol. 106, Jan-Feb 2015, pp. 139-159, ISSN 0094-5765, http://dx.doi.org/10.1016

17 'Emergency Asteroid Defence Project' < http://eadproject.com/> accessed 21 November 2019

18 'Help Defend Earth Against Asteroid Threats' <https://www.indiegogo.com/projects/help-defend-earth-againstasteroid-threats\#/> accessed 21 November 2019
} 


\subsection{Hayabusa}

On May 9th, 2003, JAXA launched the Hayabusa spacecraft to the PHA 25143 Itokawa (1998 $\left.\mathrm{SF}_{36}\right)$, with the goals of rendezvousing with the asteroid, studying it with onboard instruments, and returning samples of the asteroid material to Earth. The mission was originally designated Mu Space Engineering Spacecraft $\mathrm{C}$ (MUSES-C), but its name was subsequently changed to Hayabusa, which means "peregrine falcon" in Japanese.

Itokawa is categorised as a PHA due to its Earth MOID of 0.013 au. The asteroid is also relatively accessible for spacecraft missions, with a heliocentric orbit semi-major axis of 1.324 au, eccentricity of 0.28 , and inclination of $1.62^{\circ}$. Its absolute magnitude is 19.2 , which suggests that its approximate size should be between $250 \mathrm{~m}$ and $1,100 \mathrm{~m}$, depending on how reflective the asteroid's surface is. The Hayabusa mission discovered that Itokawa is somewhat irregularly shaped, with approximate physical dimensions of $535 \mathrm{~m} \times 294$ $\mathrm{m} \times 209 \mathrm{~m}$, and that the asteroid has an average density of $1.9 \mathrm{~g} / \mathrm{cm}^{3}$. Itokawa rotates with a period of 12.132 hours. ${ }^{19}$

Hayabusa's low-thrust solar electric xenon propulsion system brought it to rendezvous with Itokawa in September of 2005, after which the spacecraft performed station-keeping approximately $20 \mathrm{~km}$ from the asteroid. Hayabusa later moved to the asteroid's surface for sample collection by means of firing small projectiles into the asteroid's surface and then collecting the material kicked up by the impacts of those projectiles. However, the spacecraft experienced a variety of technical problems that impeded the sample collection attempt, and only a few tiny grains of asteroid material were actually collected.

Prior to the spacecraft's collection of material from Itokawa, Hayabusa had deployed a small minilander (approximately $10 \mathrm{~cm}$ in size) named MINERVA, which was intended to descend to Itokawa's surface and then utilise an internal flywheel to hop between locations on the asteroid. However, errors in deployment resulted in MINERVA instead tumbling away into space, away from Itokawa.

Hayabusa's initiation of its flight back to Earth after asteroid sample collection was delayed due to technical issues with the attitude control and propulsion systems. However, the spacecraft's mission control personnel were able to resolve those issues and the spacecraft began its journey back to Earth in April of 2007. Hayabusa reached Earth and deployed its sample return capsule on June 13th, 2010, at which time both the spacecraft and sample return capsule entered the Earth's atmosphere. The spacecraft was destroyed by atmospheric entry, as planned, while the sample return capsule performed a parachute landing within the designated landing zone in the Woomera Prohibited Area in South Australia, after which it was successfully recovered.

19 '25143 Itokawa (1998 SF36)' (Jet Propulsion Laboratory Small-Body Database Browser)

$<$ https://ssd.jpl.nasa.gov/sbdb.cgi?sstr=Itokawa> accessed 25 November 2019 
Examination of the capsule and its contents revealed that 1,500 grains of material, each approximately 10 micrometres in size, had been collected from Itokawa. Although only a tiny amount of material, this would still constitute the first return of asteroid material to Earth. Analyses of those samples suggest that Itokawa was previously part of a larger asteroid, indicate that Itokawa's surface material is approximately 8 million years old, and show that Itokawa's composition matches that of an LL chondrite meteorite (largely comprised of the minerals pyroxene and olivine, with some iron oxide). ${ }^{20}$

\subsection{Hayabusa2}

Following the success of the Hayabusa mission, JAXA developed and deployed the Hayabusa2 spacecraft to visit, study, and return samples from the PHA 162173 Ryugu (1999 JU3). Ryugu is a dark, primitive carbonaceous asteroid of the rare spectral type Cg (exhibiting characteristics of both C-type and G-type asteroids).

Ryugu is categorised as a PHA due to its Earth MOID of 0.00063 au. The asteroid is also relatively accessible for spacecraft missions, with heliocentric orbit semi-major axis of 1.189 au, eccentricity of 0.19 , and inclination of $5.88^{\circ}$. Its absolute magnitude is 19.3 , which suggests that its approximate size should be between $240 \mathrm{~m}$ and 1,070 $\mathrm{m}$, depending on how reflective the asteroid's surface is. ${ }^{21}$ The Hayabusa2 mission discovered that Ryugu has a round-ish shape featuring an equatorial bulge, with approximate physical dimensions of 1040 $\mathrm{m} \times 1020 \mathrm{~m} \times 880 \mathrm{~m}$, and that the asteroid has an average density of $1.19 \mathrm{~g} / \mathrm{cm}^{3}$, with $>50 \%$ porosity. ${ }^{22}$

The Hayabusa2 mission launched on December 3rd, 2014 and utilised its solar electric ion thruster propulsion system to reach rendezvous with Ryugu on June 27th, 2018. The spacecraft spent nearly a year and half surveying Ryugu in close proximity and then collecting surface and subsurface samples. Additionally, the spacecraft deployed three rovers to the asteroid's surface, all of which operated nominally. Hayabusa2 departed Ryugu on November 13th, 2019 and should be delivering its sample return capsule to Earth in late 2020. After the sample return, the spacecraft may then be sent on an extended mission to perform flybys of other asteroids. ${ }^{23}$

\subsection{PROCYON}

PROCYON (Proximate Object Close flyby with Optical Navigation) was a small, low-cost spacecraft co-launched with Hayabusa2 in December of 2014. PROCYON was planned to perform a flyby of the PHA (185851) $2000 \mathrm{DP}_{107}$ during 2016.

${ }^{20}$ Multiple Authors, 'Hot Topic: Hayabusa —Dust from Itokawa' Science (issue containing seven papers) (2011) 21 '162173 Ryugu (1999 JU3)' (Jet Propulsion Laboratory Small-Body Database Browser) $<$ https://ssd.jpl.nasa.gov/sbdb.cgi?sstr=Ryugu $>$ accessed 2 December 2019

${ }^{22}$ Seiichiro Watanabe, et al, 'Hayabusa2 arrives at the carbonaceous asteroid 162173 Ryugu—A spinning topshaped rubble pile' Science, 19 Apr 2019, Vol. 364, Issue 6437, pp. 268-272, DOI: 10.1126/science.aav8032

23 'Hayabusa2' (Wikipedia) <https://en.wikipedia.org/wiki/Hayabusa2> accessed 2 December 2019 
$2000 \mathrm{DP}_{107}$ is categorised as a PHA due to its Earth MOID of 0.0147 au. Its heliocentric orbit semimajor axis is $1.365 \mathrm{au}$, its eccentricity is 0.376 , and its inclination is $8.67^{\circ}$. Its absolute magnitude is 18.2 , which suggests that its approximate size should be between $400 \mathrm{~m}$ and $1,800 \mathrm{~m}$, depending on how reflective the asteroid's surface is. Characterisation via planetary radar revealed that $2000 \mathrm{DP}_{107}$ is a binary asteroid system, where the primary object in the system has dimensions of $992 \times 938 \times 964 \mathrm{~m}$, a bulk density of approximately $1.365 \mathrm{~g} / \mathrm{cm}^{3}$ (with bulk porosity likely in the range of 55--65\%), and a geometric albedo of 0.137 . Lightcurve data indicates the primary object is rotating with a period of 2.7754 hours. ${ }^{24}$ The diameter of the secondary object is approximately $300 \mathrm{~m}$, and the objects orbit one another with a 1.76-day period at a separation distance of 2,600 m. Remote observations of its spectra indicate that $2000 \mathrm{DP}_{107}$ exhibits both C-type (carbonaceous) and M-type (metallic) characteristics.

PROCYON initiated the use of its ion thruster in February of 2015 to begin its journey towards 2000 $\mathrm{DP}_{107}$. However, the ion thruster system failed in March of 2015, and contact with the spacecraft was lost in December of 2015. Prior to loss of contact, the spacecraft was able to provide observations of the hydrogen coma of comet 67P/Churyumov-Gerasimenko (the destination of ESA's Rosetta mission) and assess the rate at which the comet discharges water. This constituted the first scientific observations collected by such a small spacecraft ( $\sim 70 \mathrm{~kg}$ mass, $\sim 60 \mathrm{~cm}$ size $).{ }^{25}$ Additionally, the spacecraft was able to collect the first complete observations of Earth's hydrogen corona, enabling the discovery of its north-south symmetry. ${ }^{26}$

\subsection{DESTINY+}

DESTINY+ (Demonstration and Experiment of Space Technology for INterplanetary voYage Phaethon fLyby dUSt science) is a spacecraft mission currently under development at JAXA for a 2022 launch, with the goal of studying and understanding interplanetary dust and its origins. ${ }^{27}$ The spacecraft design utilises efficient solar electric low-thrust ion engines and demonstrates very lightweight solar panel technology. ${ }^{28}$ As part of its mission profile, DESTINY+ would perform a flyby of the PHA 3200 Phaethon (1983 TB), which has been observed to emit dust despite appearing to otherwise display asteroidal, rather than cometary, characteristics.

Phaeton is categorised as a PHA due to its Earth MOID of $0.0195 \mathrm{au}$. It occupies a rather elliptical and inclined orbit around the Sun, with heliocentric orbit semi-major axis of 1.271 au, eccentricity of 0.89 , and

\footnotetext{
24 '185851 (2000 DP107)' (Jet Propulsion Laboratory Small-Body Database Browser) $<$ https://ssd.jpl.nasa.gov/sbdb.cgi?sstr=2000DP107> accessed 25 November 2019

25 'Micro spacecraft investigates cometary water mystery' (National Astronomical Observatory of Japan, 24 Jan 2017) <https://www.nao.ac.jp/en/news/science/2017/20170124-procyon.html> accessed 22 November 2019

${ }^{26}$ Shingo Kameda, et al, 'Ecliptic North-South Symmetry of Hydrogen Geocorona', Geophysical Research Letters, Vol 44, Issue 23, 16 December 2017, Pages 11,706-11,712

27 'DESTINY+' (JAXA)<https://destiny.isas.jaxa.jp/> accessed 25 November 2019

${ }^{28}$ Hiroyuki Toyota, et al, 'DESTINY+: Deep Space Exploration Technology Demonstrator and Explorer to Asteroid 3200 Phaethon', Low-Cost Planetary Missions Conference, Pasadena, CA, August 15 - 17, 2017, $<$ http://www.lcpm12.org/wp-content/uploads/2017/08/1415-1435-Toyota.pdf > accessed 25 November 2019
} 
inclination of $22.26^{\circ}$. Its absolute magnitude is 14.6 , which suggests that its approximate size should be between 2,100 $\mathrm{m}$ and 9,200 $\mathrm{m}$, depending on how reflective the asteroid's surface is. Arecibo radar observations show the object's size to be about $6,250 \mathrm{~m} \cdot{ }^{29}$ Infrared Astronomical Satellite (IRAS) observations indicate the object's geometric albedo is 0.1066 , and combining that with the aforementioned absolute magnitude yields an estimated size of $4,890 \mathrm{~m}$, which is within approximately $20 \%$ of the actual object size as assessed via radar. The $4,890 \mathrm{~m}$ size estimate based on the absolute magnitude and albedo is much closer to the results of thermophysical modelling, which estimated the effective diameter of the object to be 5,100 +/- $200 \mathrm{~m} .{ }^{30}$ Lightcurve observations of Phaeton indicate that it rotates with a period of about 3.6 hours. ${ }^{31}$

Unusual for an object with asteroidal properties, Phaeton is the parent body of the mid-December Geminids meteor shower. It is categorised as an NEA yet occupies a rather elliptical and inclined orbit that is more reminiscent of a cometary orbit. As well, at times the object has appeared to be ejecting dust and/or exhibiting a dust tail. Thus, Phaeton seems to have a mixture of asteroidal and cometary characteristics, and is, therefore, an interesting object for more detailed study.

6.5 The Japan Spaceguard Association

The Japan Spaceguard Association (JGSA) is a Specified Nonprofit Corporation in Japan whose goal is to observe and study NEOs for the purpose of supporting defence against NEO impacts on Earth. Towards this end, the JGSA operates the Bisei Spaceguard Center (BSGC), which is owned by the Japan Space Forum and located near the town of Bisei (Oda District, Okayama Prefecture) in western Japan. ${ }^{32}$

The BSGC is an observatory facility constructed during 1999--2000 that carries out the Bisei Asteroid Tracking Telescope for Rapid Survey (BATTeRS), which has discovered 440 minor planets as of October 2019. The observatory operates a 1-meter telescope for object search, and a 0.5-meter telescope for follow-up observations. $^{33}$

\section{Luxembourg Government}

\footnotetext{
${ }^{29}$ Patrick Taylor, et al, 'Arecibo Radar Observations of near-Earth asteroid (3200) Phaethon during the 2017 apparition' Planetary and Space Science, Vol 167, March 2019, pages 1-8, $<$ https://doi.org/10.1016/j.pss.2019.01.009>

${ }^{30}$ Josef Hanuš, et al, 'Near-Earth asteroid (3200) Phaethon: Characterization of its orbit, spin state, and thermophysical parameters', Astronomy \& Astrophysics, Vol 592, August 2016, A34, $<$ https://doi.org/10.1051/0004-6361/201628666>

31 '3200 Phaethon (1983 TB)' (Jet Propulsion Laboratory Small-Body Database Browser) $<$ https://ssd.jpl.nasa.gov/sbdb.cgi?sstr=Phaethon> accessed 25 November 2019

32 'Japan Spaceguard Association' (Wikipedia, 23 September 2019) $<$ https://en.wikipedia.org/wiki/Japan_Spaceguard_Association> accessed 25 November 2019

33 'Bisei Spaceguard Center' (Wikipedia, 17 October 2019) $<$ https://en.wikipedia.org/wiki/Bisei_Spaceguard_Center> accessed 25 November 2019
} 
In February of 2016, the Luxembourg government announced its intentions to begin creating a legal framework for the mining of resources in space, which, of course, includes the mining of asteroids. Although asteroid mining is not specifically oriented towards planetary defence, the activities necessary to support and conduct asteroid mining are very synergistic with planetary defence objectives. Asteroid mining activities may include: telescopically searching for asteroids; characterising the orbital and physical properties of asteroids; sending robotic spacecraft to asteroids for resource prospecting (which would collect most, if not all, of the data about the asteroid that would be desired for planetary defence related assessments); designing/testing systems for manipulating asteroid material; and, eventually, sending actual mining missions to asteroids. Such activities would accelerate the discovery of Earth-impacting NEOs before their Earth impact dates, and help advance technologies needed for characterising and interacting with NEOs, all of which is highly relevant to planetary defence.

The Luxembourg Space Agency (LSA) continues to operate its "SpaceResources.lu Initiative," the goals of which include ensuring "... that space resources explored under its jurisdiction serve a peaceful purpose, are gathered and used in a sustainable manner compatible with international law and for the benefit of humankind." 34 As incentives to commercial entities pursuing related activities, Luxembourg offered financial investments in research and development work, as well as tax credits. An agreement was also made with the U.S.-based company Deep Space Industries, Inc. (DSI) whereby Luxembourg would co-fund DSI's development of asteroid rendezvous spacecraft missions, for purposes of asteroid rendezvous technology demonstration as a precursor to resource prospecting and eventual asteroid mining. ${ }^{35}$

However, those missions have yet to be brought to fruition. While LSA maintains its intentions to pursue asteroid resource utilisation efforts, it is worth noting that the U.S. asteroid mining companies $\operatorname{DSI}^{36}$ and Planetary Resources, Inc. ${ }^{37}$ have both since been purchased by other companies for purposes other than asteroid mining. As such, while interest in asteroid mining remains, the status and future of such missions is unclear.

\section{Russia}

\footnotetext{
34 'Spaceresources.lu initiative' (Luxembourg Space Agency) <https://space-agency.public.lu/en/space-resources/theinitiative.html> accessed 2 December 2019

35 Peter B. de Selding, 'Luxembourg invests to become the "Silicon Valley of space resource mining"' (SpaceNews, 3 June 2016) <https://spacenews.com/luxembourg-invests-to-become-the-silicon-valley-of-space-resource-mining/> accessed 2 December 2019

${ }^{36}$ Jeff Foust, 'Deep Space Industries acquired by Bradford Space' (SpaceNews, 2 January 2019)

$<$ https://spacenews.com/deep-space-industries-acquired-by-bradford-space/> accessed 2 December 2019

37 Jeff Foust, 'Asteroid mining company Planetary Resources acquired by blockchain firm' (SpaceNews, 31 October 2018) $<$ https://spacenews.com/asteroid-mining-company-planetary-resources-acquired-by-blockchain-firm/> accessed 2 December 2019
} 
Russia has the distinction of being the location of the three most significant asteroid impacts in recent history: The Tunguska event in Siberia in $1908,{ }^{38}$ the Chelyabinsk meteor in $2013^{39}$, and the Kamchatka meteor in $2018 .{ }^{40}$ However, despite this, Russia's involvement in planetary defence and related activities has been limited to date. Thus far, there has been some Russian participation in the International Asteroid Warning Network (IAWN), and the Russian Academy of Sciences has expressed intentions to develop national programs aimed at planetary defence research and development.

\section{Conclusion}

Herein we have surveyed some of the more notable activities related to planetary defence taking place around the world beyond the efforts at the United Nations, NASA, and ESA. Of these, the most significant are the Hayabusa and Hayabusa2 asteroid sample return missions flown by JAXA, followed by the Chang'e 2 flyby of Toutatis performed by CNSA. Thus, the Japanese and Chinese space agencies are currently the two national space agencies most active in planetary defence related endeavours, besides NASA and ESA. In terms of both flown and planned spacecraft missions, JAXA is the most active overall.

It is clear that planetary defence and related activities are considered to be important by various organisations the world over. Additional missions to asteroids are in development, and research programs are proceeding apace. Assuming the trend of increasing activity levels continues, we can expect to see planetary defence related activities beyond those at NASA and ESA continue to expand in the years to come. We can also expect to see increasing international collaboration on planetary defence and related topics. These are certainly desirable outcomes, as the opportunities presented by asteroids and comets may benefit our entire species and the hazards presented by such objects pose a threat to all the people and nations of the world.

${ }^{38}$ Lorien F. Wheeler, Donovan Mathias, 'Probabilistic assessment of Tunguska-scale asteroid impacts', Icarus 327 (2019)

${ }^{39}$ Olga P. Popova, et al, 'Chelyabinsk Airburst, Damage Assessment, Meteorite Recovery, and Characterization', Science 342 (2013)

${ }^{40}$ Leonard David, 'Huge Meteor Explosion a Wake-Up Call for Planetary Defense' (Scientific American, 21 March 2019) <https://www.scientificamerican.com/article/huge-meteor-explosion-a-wake-up-call-for-planetary-defense/> accessed 26 December 2019 